\title{
Evolutionary analysis of the SUB1 locus across the Oryza genomes
}

Railson Schreinert dos Santos ${ }^{1,2}$, Daniel da Rosa Farias², Camila Pegoraro², Cesar Valmor Rombaldi ${ }^{1}$, Takeshi Fukao ${ }^{3}$, Rod A. Wing ${ }^{4}$ and Antonio Costa de Oliveira ${ }^{1,2^{*}}$

\begin{abstract}
Background: Tolerance to complete submergence is recognized in a limited number of Asian rice (Oryza sativa L.) varieties, most of which contain submergence-inducible SUBTA on the polygenic SUBMERGENCE-1 (SUB1) locus. It has been shown that the SUB1 locus encodes two Ethylene-Responsive Factor (ERF) genes, SUB1B and SUB1C, in all $O$. sativa varieties. These genes were also found in O rufipogon and O nivara, wild relatives of O. sativa. However, detailed analysis of the polygenic locus in other Oryza species has not yet been made.

Findings: Chromosomal location, phylogenetic, and gene structure analyses have revealed that the SUB1 locus is conserved in the long arm of chromosome 9 in most Oryza species. We also show that the SUB1A-like gene of $O$. nivara is on chromosome 1 and that Leersia perrieri, a grass-tolerant to deep-flooding, presents three ERF genes in the SUB1 locus.

Conclusion: We provide here a deeper insight into the evolutionary origin and variation of the SUB1 locus and raise the possibility that an association of these genes with flooding tolerance in L. perrieri may exist.
\end{abstract}

Keywords: IOMAP, Oryza, Leersia perrieri, Submergence tolerance, SUB1 genes

\section{Findings}

Abiotic stresses such as flooding can prevent plants from attaining their full genetic potential for growth and reproduction. In Asia, the leading producers of rice (Oryza sativa L.), many flood-prone lands are used for rice production, reason why so many studies are developed aiming to understand and overcome flooding response mechanisms.

A major quantitative trait locus (QTL) called SUBMERGENCE-1 (SUB1) is responsible for conferring submergence tolerance to the rice landrace Flood Resistant 13A (FR13A). Detailed sequence analysis revealed that the locus encodes a variable cluster of up to three Ethylene-Responsive Factors (ERF): SUB1A, SUB1B and SUB1C (Fukao et al., 2006; Xu et al., 2006).

All surveyed $O$. sativa varieties contain $S U B 1 B$ and $S U B 1 C$, whereas SUB1A is present only in a limited

\footnotetext{
*Correspondence: acostol@cgfufpel.org

${ }^{1}$ Technology Development Center (CDTec), Universidade Federal de Pelotas, Pelotas, Brazil

${ }^{2}$ Plant Genomics and Breeding Center (CGF), Universidade Federal de

Pelotas, Pelotas, Brazil

Full list of author information is available at the end of the article
}

number of accessions. Genotypes containing submergenceinducible SUB1A restricts underwater ethylene production, which maintains mRNA and protein accumulation of gibberellic acid (GA) signaling repressors during submergence (Fukao and Bailey-Serres, 2008; Fukao et al., 2006; Singh et al., 2014). SUB1A-mediated inhibition of GA responsiveness consequently represses genes required for starch and sucrose catabolism. This strategy characterized by reduced plant elongation in order to preserve the sugars needed for regrowth when water recedes is called 'quiescence strategy' and it is being very useful in plant breeding (Neeraja et al., 2007; Fukao and Bailey-Serres, 2008; Fukao et al., 2006; Septiningsih et al., 2009; Septiningsih et al., 2013; Iftekharuddaula et al., 2015).

Wild rice species generally lack agriculturally important traits. However, they carry many desirable genes that have been lost in cultivated rice. Here, we report that previous analyses made by Fukao et al. (2009) and Niroula et al. (2012) are now extended across large part of Oryza phylogeny using sequencing data from the International Oryza Map Alignment Project (IOMAP) consortium (Stein et al: Sequence of 11 rice-related 
species unveils the Oryza pan-genome and the origin of genetic innovation, submitted; Jacquemin et al., 2013): $O$. sativa $\mathrm{L}$. ssp indica (AA), O. sativa L. ssp japonica (AA), O. rufipogon Griff. (AA), O. nivara Sharma et Shastry (AA), O. glumaepatula Steud. (AA), O. glaberrima S. (AA), O. barthii A. Chev. (AA), O. meridionalis Ng. (AA), O. punctata Kotschy ex Steud. (BB), and $O$. brachyantha Chev. et Roehr (FF).

Our genomic analysis identified 58 SUB1-like genes (Additional file 1: Table S1). The alignment of the AP2 domains with the signature amino acids alanine and aspartic acid at positions 13 and 18, respectively, of the AP2 domain, a characteristic of the B2 subgroup of ERF proteins, can be seen in Additional file 2: Figure S1.
Of nine species, six of them such as O. rufipogon (AA), O. nivara (AA), O. glumaepatula (AA), O. glaberrima (AA), O. sativa (AA), and O. punctata (BB) contained both $S U B 1 B$ - and $S U B 1 C$-like genes side by side on chromosome 9 (Chr 9), as shown in Fig. 1a and b, which is consistent with the observations in multiple accessions of O. sativa (AA) (Singh et al., 2010; Xu et al., 2006). According to its position in the genome and to the proximity between these genes we first divided these SUB1-like genes into two large supergroups: "Other SUB1-like proteins" and "SUB1 loci", as seen in Fig. 1a.

We further divided the supergroup called "Other SUB1-like proteins", which is formed by longer proteins, into three groups: "SUB1-like Chr 2" (SUB1chr2),

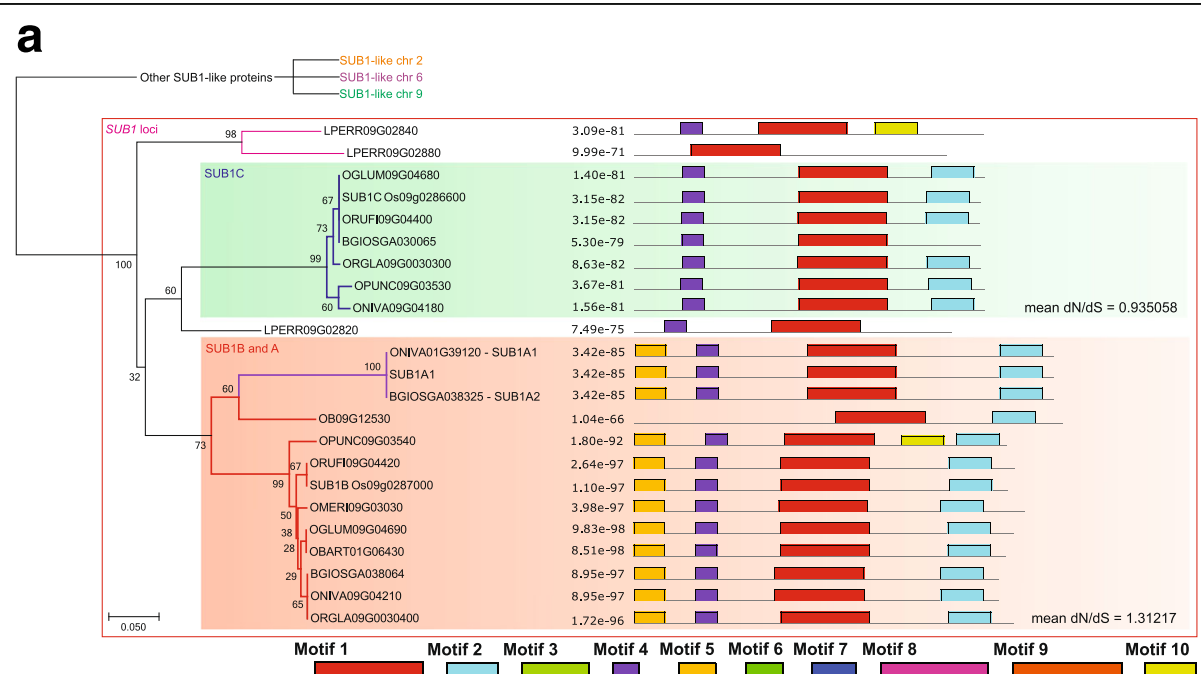

b

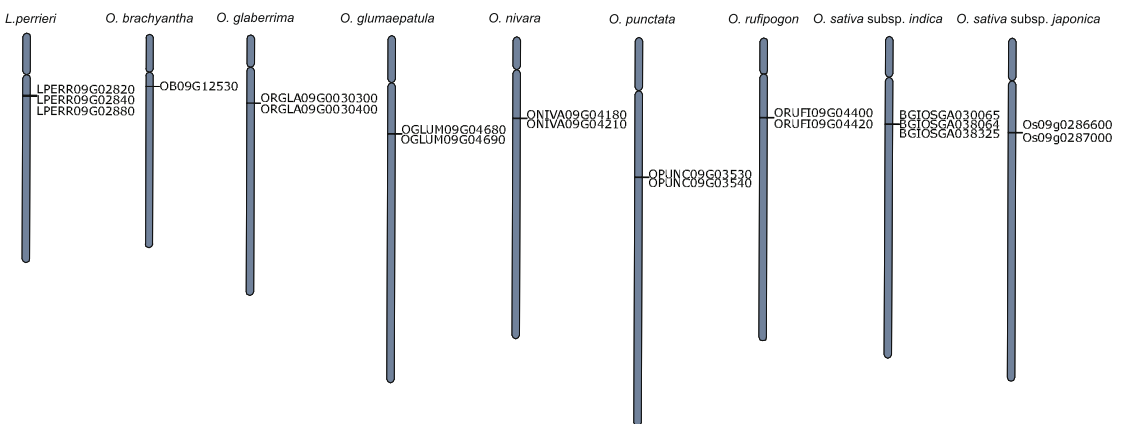

Chr 9

C

\begin{tabular}{ll}
\multicolumn{1}{|c}{13} & 18 \\
${ } }$ & YHGIRQRPWGRWSSEIRDPVKGVRLWLGTFDTAVEAALAYDAEARRIHGWKARTNF \\
ONIVA01G39120 & YHGIRQRPWGRWSSEIRDPVKGVRLWLGTFDTAVEAALAYDAEARRIHGWKARTNF \\
SUB1A1 & YHGIRQRPWGRWSSEIRDPVKGVRLWLGTFDTAVEAALAYDAEARRIHGWKARTNF
\end{tabular}

Fig. 1 SUB1 analyses across the Oryza genomes. a Phylogenetic tree generated from the protein alignment of SUB1-like genes identified of all species analyzed, genes in red are SUB1B-like genes and green are SUB1C-like. On the right side of the tree, amino acid motifs are represented. Full version and motif logos are available in Additional file 3: Figure S2 and Additional file 11: Figure S8. b Chromosomal location of the SUB1-like loci in each Oryza genome (chromosomes 1 and 9). Full version available in Additional file 4: Figure S3. c Alignment of the AP2 domains of SUB1A-like genes in rice relatives 
"SUB1-like Chr 6" (SUB1chr6) and "SUB1-like Chr 9" (SUB1chr9) (Additional file 3: Figure S2). SUB1chr2 consists of genes on Chr 2, while SUB1chr6 group consists of genes at the Chr 6 with a deletion of motif 10, and an inverted region, with motif 7 after motif 2 . The third group, SUB1chr9, is present on Chr 9 and genes of this group possess the motif 9 preceding motif 3. O. glumaepatula showed the most distinct arrangement of motifs with a deletion covering motifs 5 and 4 which made the gene much shorter in this species. O. brachyantha presented a gene on SUB1chr9 that resembles those grouped as SUB1chr2.

The OsERF\#70 (Os02g0782700), which is the SUB1chr2 gene found in $O$. sativa is already known to interact with a Myc transcription factor called OsBP-5, coregulating the expression of the waxy gene (Zhu et al., 2003). Such information shows that the genes contained in the supergroup "Other SUB1-like genes" have the potential to interact with Myc factors. The similarities of these longer genes with those found in the SUB1 locus should be better studied in order to analyze their significance in altering plant tolerance to stresses and/or other important traits that may bring interesting insights about ERF evolution or that may be of interest of breeders.

The second large supergroup, which we called "SUB1 loci" (Fig. 1a), is formed by shorter genes, on the long arm of Chr 9, near the centromere (Fig. 1b). Most of these genes are positioned in a similar way as the SUB1 locus described by $\mathrm{Xu}$ et al. (2006). Figure $1 \mathrm{~b}$ and Additional file 4: Figure S3 show the positions of these genes on chromosomes of different genomes.

Interestingly, we also found $S U B 1 A$ - and $S U B 1 B$-like genes on Chr 1 of O. nivara (ONIVA01G39120) and O. barthii (OBART01G06430), respectively. To identify possible translocation events related to these specific loci we aligned regions corresponding to $50 \mathrm{~Kb}$ upstream and $50 \mathrm{~Kb}$ downstream of ONIVA01G39120 and OBART01G06430 to SUB1 loci (Chr 9) of each Oryza species. The alignments show no similar regions when SUB1 loci are compared to ONIVA01G39120, nevertheless we can identify an orthologous region $(\sim 8.5 \mathrm{~Kb})$ when SUB1 loci of all genome AA species are compared to OBART01G06430 (Additional file 5: Figure S4). The region was searched for the presence of transposable elements (TEs) that were possibly involved with this event, but no clear mechanism involving TEs was detected.

It is also interesting to highlight that the genomes of $O$. meridionalis (AA), O. barthii (AA) and O. brachyantha (FF) encoded only one SUB1-like gene, all of them more related to $S U B 1 B$ than to $S U B 1 C$.

Three genes closely related to SUB1 locus were found in Chr 9 of Leersia perrieri (A. Camus) Launert. (LPERR09G02820, LPERR09G02840 and LPERR09G02880) demonstrating the presence of duplication events in the onset of $S U B 1 B$ and $S U B 1 C$, which occurred after the divergence of Oryzeae from other grasses. This duplication event seems to have occurred before the speciation of $L$. perrieri.

SUB1A-like genes were not detected in the genomes of these rice relatives, except for O. nivara (Fig. 1c). An alignment of the gene found by Niroula et al. (2012) (cultivar IRGC:80507), ONIVA01G39120 (cultivar IRGC:100897) and original SUB1A-1 demonstrates that, despite differences in the $\mathrm{C}$-terminal region, the two $O$. nivara sequences are probably variants (alleles) from the same locus. $O$. brachyantha also presented a gene that was similar to SUB1 (OB09G12530), but the absence of a serine at position 13 , and other differences along their sequence prevented its classification as a $S U B 1 A$-like gene. It is interesting to notice that the SUB1A-like genes found in O. nivara (ONIVA01G39120) and O. barthii (OBART01G06430), were not on Chr 9, but on Chr 1.

Constitutive and conditional expression of SUB1A significantly increases tolerance to complete submergence in O. sativa (Fukao et al., 2006; Xu et al., 2006). Detailed genetic survey demonstrated that submergence-tolerant accessions of $O$. sativa, $O$. nivara and O. rufipogon possess SUB1A-1 which is highly induced by submergence, suggesting that SUB1A arose via tandem duplication prior to rice domestication (Niroula et al., 2012; Singh et al., 2010). In this study, we surveyed the entire genome sequences of nine rice relatives to identify orthologs of $S U B 1 A$ as well as SUB1B and SUB1C.

Chromosomal location of the Chr 9 SUB1 locus was highly conserved in O. nivara, O. rufipogon, O. glumaepatula and O. glaberrima which had a locus deviation smaller than $2.0 \mathrm{Mb}$. The deviation of $O$. punctata, which also had a similar conserved structure in the long arm of Chr 9, was a little larger reaching $3.06 \mathrm{Mb}$ (Fig. 1b).

To estimate how natural selection is acting on the coding sequences of $S U B 1 C$ - and $S U B 1 B$-like genes, the ratio of synonymous/non-synonymous mutations (dN/dS) was calculated (Fig. 1a and Additional file 6: Figure S5). The mean $\mathrm{dN} / \mathrm{dS}$ is 1.312 (positive selection) for $S U B 1 B$ - and 0.935 (negative selection) for SUB1C-like genes. The freedom that SUB1B-like genes have to be modified may have been essential to the emergence of $S U B 1 A$-like genes, which are extremely close to $S U B 1 B$ genes.

The analysis of the upstream sequences $(-1,500 \mathrm{bps})$ of SUB1A-like genes shows that the gene found in $O$. nivara must have a similar regulation to the SUB1A-1 allele responsible for tolerance to submergence in $O$. sativa (Additional file 7: Figure S6). The transcription factor binding sites (TFBSs) found in the upstream region of ONIVA01G39120 (Additional file 8: Table S2), 
together with the location of polymorphisms found when comparing SUB1A-1 and SUB1A-2 alleles from $O$. sativa (Additional file 9: Figure S6) shows that diverse transcription factors, among them WRKY and ERF, may be important regulators of this gene in submergence conditions. This conclusion is based on the fact that binding sites for these TFs were detected in families where differences between the promoter regions of SUB1A-1 (upregulated by submergence) and SUB1A-2 (non-upregulated by submergence) alleles were found. Now we should further investigate the possibly tolerant phenotype resulting from the introgression of ONIVA01G39120 into O. sativa elite cultivars and how is it regulated since this constitutes a very relevant information to breeders.

Integration of genome sequences with RNAseq data allowed us to construct gene structure models of SUB1-like genes isolated from these rice relatives (Additional file 10: Figure S7). Gene structure analysis revealed the presence of an intron inside the coding sequence (CDS) of SUB1B-like genes, except for $O$. glaberrima, $O$. rufipogon and $O$. glumaepatula.

SUB1C alleles of O. sativa contain an intron in the 3' untranslated region (UTR). However, our gene structure analysis based on RNAseq data did not detect 3' UTR sequences of SUB1C-like genes (Additional file 10: Figure S7). Therefore, the intron in the 3' UTR region of SUB1C-like genes was sought within $1.5 \mathrm{~kb}$ downstream of the stop codon in these genes and regions highly similar to the O. sativa intron were found in ORUFI09G04400, ONIVA09G04180, OGLUM09G04680, OGLAB09G030300 and OPUNC09G03530 indicating that most SUB1C-like genes identified in Oryza species possess traces of an intron in their terminal region (Additional file 11: Table S3).

In summary, here we present SUB1-like genes identified in wild rice species through genome-wide sequence analysis. Our chromosomal location, phylogenetic, and gene structure analyses have revealed that the SUB1 locus is conserved in the long arm of Chr 9 in most Oryza species with AA-, BB- and FF-genomes. We also showed that the SUB1A-like gene of O. nivara is on Chr 1 and that L. perrieri, a grass-tolerant deep-flooding, presents three $E R F$ genes in SUB1 locus, similar to that found in flooding tolerant rice. This finding raises the possibility that an association of this gene with flooding tolerance in $L$. perrieri exists. Along with genes found in Chr 1, SUB1-like genes found in Chr 2 and Chr 6 represent some new interesting information. These genes should now be further investigated in order to check their biological and agricultural significance. Allelic survey of diverse ecotypes in wild rice species is necessary to determine the evolutionary origin and biological significance of SUB1A in the genus Oryza (Additional file 12: Table S4, Additional file 13: Table S5 and Additional file 14).

\section{Additional files}

Additional file 1: Table S1. SUB1-like genes identified in Oryza species. (XLSX $22 \mathrm{~kb}$ )

Additional file 2: Figure S1. Alignment of the AP2 domains of SUB1like genes in rice relatives. Signature amino acids specific to B2 subgroup of ERF proteins are highlighted. (PDF $403 \mathrm{~kb}$ )

Additional file 3: Figure S2. Phylogenetic tree generated from the protein alignment of SUB1-like genes identified of all species analyzed, genes in red are SUB1B-like genes and green are SUB1C-like. On the right side of the tree, amino acid motifs are represented. Motif logos are available in Additional file 11: Figure S8. (PDF 1078 kb)

Additional file 4: Figure S3. Chromosomal location of the SUB1-like loci in each Oryza genome (chromosomes 1 and 9). (PDF $457 \mathrm{~kb}$ )

Additional file 5: Figure S4. Alignment of OBART01G06430 genomic region (first line) to SUB1 loci (Chr 9) regions of Oryza species of AA genome. Same colors represent collinear blocks between genomes. (PDF 174 kb)

Additional file 6: Figure S5. Phylogenetic tree, motifs and evolutionary pressures on proteins quantified by the ratio of substitution rates at nonsynonymous and synonymous sites (dN/dS) for SUB1B-like and SUB1C-like genes. (PDF 582 kb)

Additional file 7: Figure S6. Alignment of the promoter region (1,500 bps upstream) of SUB1A-like genes, showing polymorphisms found when comparing SUB1A-1 and SUB1A-2 alleles. (PDF $2215 \mathrm{~kb}$ )

Additional file 8: Table S2. Transcription Factor Binding Sites (TFBSs) in the promoter regions (-1,500 bps upstream) of SUB1A-like gene of $O$. nivara. Regions polymorphic when comparing (XLSX $212 \mathrm{~kb}$ )

Additional file 9: Figure S7. Structure of the SUB1-like genes in each of the different species. (PDF $399 \mathrm{~kb}$ )

Additional file 10: Table S3. BLAST analysis searching the $3^{\prime} U T R$ intron in SUB1C-like genes. (XLSX 12 kb)

Additional file 11: Figure S8. Motifs found on the analysis of the amino acid sequences of SUB1-like genes. (PDF $416 \mathrm{~kb}$ )

Additional file 12: Table S4. Genes with e-value below $1 e^{-25}$ found by BLAST. (XLSX $21 \mathrm{~kb})$

Additional file 13: Table S5. Gene structure information (General feature format) about each of the analyzed genes.. (XLSX $239 \mathrm{~kb}$ )

Additional file 14: Methods. (DOCX $24 \mathrm{~kb}$ )

\section{Abbreviations}

CDS: Coding sequence; dN/dS: Ratio of synonymous/non-synonymous mutations; ERF: Ethylene-Responsive Factor; FR13A: Flood Resistant 13A; GA: Gibberellic acid; IOMAP: International Oryza Map Alignment Project; QTL: Quantitative trait locus; SUB1: SUBMERGENCE-1; UTR: Untranslated region

\section{Acknowledgments}

We would like to thank the Virginia Agricultural Experimental Station and the Hatch Program of the National Institute of Food and Agriculture, U.S. Department of Agriculture. No conflict of interest declared.

\section{Funding}

This work was supported by the Brazilian Ministry of Science and Technology, National Counsel of Technological and Scientific Development (CNPq) (477083/ 2013-7); Coordination for the Improvement of Higher Education Personnel (CAPES) (2880/2011) and RS State Foundation for Research Support (FAPERGS) (2232-2551/14-6).

Availability of data and materials

Methods (Additional file 14: Methods) and other additional files are available at Rice Online.

\section{Author contributions}

RSS. and DRF. conducted the major bioinformatics analyses, data interpretation and wrote the manuscript. CP, CVR, TK., RAW. contributed to 
performing the research and revising the manuscript, and ACO. conceived the study and supervised the research. All authors read and approved the final manuscript.

\title{
Competing interests
}

The authors declare that they have no competing interests.

\section{Consent for publication}

\author{
Not applicable.
}

\section{Author details}

${ }^{1}$ Technology Development Center (CDTec), Universidade Federal de Pelotas, Pelotas, Brazil. ${ }^{2}$ Plant Genomics and Breeding Center (CGF), Universidade Federal de Pelotas, Pelotas, Brazil. ${ }^{3}$ Crop and Soil Environmental Sciences, Virginia Tech, Blacksburg, USA. ${ }^{4}$ The School of Plant Sciences, Ecology \& Evolutionary Biology, Arizona Genomics Institute, Tucson, USA.

Received: 6 August 2016 Accepted: 20 December 2016

Published online: 07 February 2017

\section{References}

Fukao T, Bailey-Serres J (2008) Submergence tolerance conferred by Sub1A is mediated by SLR1 and SLRL1 restriction of gibberellin responses in rice. Proc Natl Acad Sci U S A 105:16814-16819. doi:10.1073/pnas.0807821105

Fukao T, Harris T, Bailey-Serres J (2009) Evolutionary analysis of the Sub1 gene cluster that confers submergence tolerance to domesticated rice. Ann Bot 103:143-150. doi:10.1093/aob/mcn172

Fukao T, Xu K, Ronald PC, Bailey-Serres J (2006) A variable cluster of ethylene response factor-like genes regulates metabolic and developmental acclimation responses to submergence in rice. Plant Cell 18:2021-2034. doi: 10.1105/tpc. 106.043000

Iftekharuddaula KM, Ahmed HU, Ghosal S, Moni ZR, Amin A, Ali MS (2015) Development of New Submergence Tolerant Rice Variety for Bangladesh Using Marker-Assisted Backcrossing. Rice Sci 22:16-26. doi:10.1016/j.rsci.2015. 05.003

Jacquemin J, Bhatia D, Singh K, Wing RA (2013) The International Oryza Map Alignment Project: development of a genus-wide comparative genomics platform to help solve the 9 billion-people question. Curr Opin Plant Biol 16: 147-156. doi:10.1016/j.pbi.2013.02.014

Neeraja CN, Maghirang-Rodriguez R, Pamplona A, Heuer S, Collard BC, Septiningsih EM, Vergara G, Sanchez D, Xu K, Ismail AM, Mackill DJ (2007) A marker-assisted backcross approach for developing submergence-tolerant rice cultivars. Theor Appl Genet 115:767-776. doi:10.1007/s00122-007-0607-0

Niroula RK, Pucciariello C, Ho VT, Novi G, Fukao T, Perata P (2012) SUB1Adependent and -independent mechanisms are involved in the flooding tolerance of wild rice species. Plant J 72:282-293. doi:10.1111/j.1365-313X. 2012.05078.x

Septiningsih EM, Pamplona AM, Sanchez DL, Neeraja CN, Vergara GV, Heuer S, Ismail AM, Mackill DJ (2009) Development of submergence-tolerant rice cultivars: the Sub1 locus and beyond. Ann Bot 103:151-160. doi:10.1093/aob/ men206

Septiningsih EM, Ignacio JCl, Sendon PMD, Sanchez DL, Ismail AM, Mackill DJ (2013) QTL mapping and confirmation for tolerance of anaerobic conditions during germination derived from the rice landrace Ma-Zhan Red. Theor Appl Genet 126:1357-1366. doi:10.1007/s00122-013-2057-1

Singh N, Dang TT, Vergara GV, Pandey DM, Sanchez D, Neeraja CN, Septiningsih EM, Mendioro M, Tecson-Mendoza EM, Ismail AM et al (2010) Molecular marker survey and expression analyses of the rice submergence-tolerance gene SUB1A. Theor Appl Genet 121:1441-1453. doi:10.1007/s00122-010-1400-z

Singh S, Mackill DJ, Ismail AM (2014) Physiological basis of tolerance to complete submergence in rice involves genetic factors in addition to the SUB1 gene. AoB Plants 6. doi: 10.1093/aobpla/plu060

Xu K, Xu X, Fukao T, Canlas P, Maghirang-Rodriguez R, Heuer S, Ismail AM, BaileySerres J, Ronald PC, Mackill DJ (2006) Sub1A is an ethylene-response-factorlike gene that confers submergence tolerance to rice. Nature 442:705-708. doi:10.1038/nature04920

Zhu Y, Cai XL, Wang ZY, Hong MM (2003) An interaction between a MYC protein and an EREBP protein is involved in transcriptional regulation of the rice $\mathrm{Wx}$ gene. J Biol Chem 278:47803-47811

\section{Submit your manuscript to a SpringerOpen ${ }^{\circ}$ journal and benefit from:}

- Convenient online submission

- Rigorous peer review

- Immediate publication on acceptance

- Open access: articles freely available online

- High visibility within the field

- Retaining the copyright to your article 